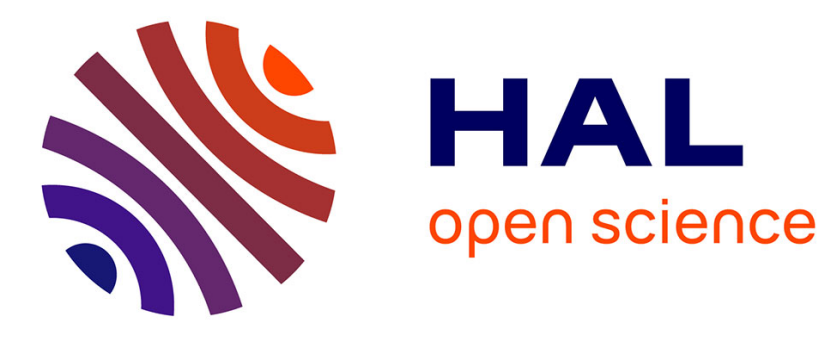

\title{
Analysis of Non-Linear Soft Thin Interfaces
}

Frédéric Lebon, Raffaella Rizzoni, Sylvie Ronel-Idrissi, Christian Licht

\section{To cite this version:}

Frédéric Lebon, Raffaella Rizzoni, Sylvie Ronel-Idrissi, Christian Licht. Analysis of Non-Linear Soft Thin Interfaces. The Sixth International Conference on Computational Structures Technology, Sep 2002, Prague, Czech Republic. 10.4203/ccp.75.65 . hal-01803575

\section{HAL Id: hal-01803575 https://hal.science/hal-01803575}

Submitted on 30 May 2018

HAL is a multi-disciplinary open access archive for the deposit and dissemination of scientific research documents, whether they are published or not. The documents may come from teaching and research institutions in France or abroad, or from public or private research centers.
L'archive ouverte pluridisciplinaire HAL, est destinée au dépôt et à la diffusion de documents scientifiques de niveau recherche, publiés ou non, émanant des établissements d'enseignement et de recherche français ou étrangers, des laboratoires publics ou privés. 


\title{
Analysis of non-linear soft thin interfaces
}

F. Lebon*, R. Rizzoni**, S. Ronel-Idrissi* and C. Licht***

*Laboratoire Mécanique Matériaux Structures, Université Claude Bernard Lyon 1, 82, Bd Niels Bohr, 69622 Villeurbanne Cedex, France

** Dipartimento di Ingegneria, Università di Ferrara, Via Saragat 1, 44100 Ferrara, Italy

*** Laboratoire de Mécanique et Génie Civil, Université Montpellier 2, Pl. E. Bataillon, 34095 Montpellier Cedex 5, France

\begin{abstract}
This paper shows how to model thin layers and interfaces by asymptotic techniques. Some behaviors are treated: visco-elasticity (Maxwell, Kelvin-Voigt, Norton), MohrCoulomb non-associated elasto-plasticity, non-monotone relationship in the strainstress diagram and contact conditions between the adhesive and the adherents. Numerical validations and algorithms are proposed and presented.
\end{abstract}

Keywords: Interface laws, asymptotic study, elasto-plasticity, non-monotone behavior, finite elements. 


\section{Introduction}

Contact and interface mechanics intervenes more and more often in computational structures. To have reliable tools to size more and more complex systems, it is indispensable to take into account in a precise way the connections between the various solids of the structure. This consideration can be very complex because of the strongly non-linear character and possibly imperfect and very localized of these connections. For examples, in the modelling of the processes of metal forming, consideration of the friction is necessary because it pilots the whole process; the modelling of the mortar in the case of masonry structures is necessary to estimate in a precise way the mechanical characteristics of the structures, their risks of ruin and collapse, ...

The purpose of this lecture is to show how it is possible to obtain families of interface laws from the mechanical behaviour of thin layers. The consideration of bonded joints in real structures can lead from a numerical point of view to problems of too large sizes, especially if these joints have a non-linear behaviour [1]. From a general way, one is going to consider joints of weak thickness and weak rigidity with regard to those of the substrata. One has then to deal with problems taking into account at least two small parameters (the thickness, the rigidity). An asymptotic study (a micromacro passage), completed by numerical calculations, leads to so-called "asymptotic contact laws" who allow describing the (macroscopic) mechanical behaviour of the interfaces (see references in [1]).

We consider in this lecture various kinds of non-linear behaviour for the thin layers:

- visco-elasticity (Maxwell, Kervin-Voigt or Norton type): one explicits the results expressed in [2];

- contact conditions of Coulomb and Signorini type between the adhesive and the adherents [3];

- non-associated elasto-plastic (Mohr-Coulomb type);

- non-monotone relationship in the strain-stress diagram.

One can show in the first and third cases, that the interface laws obtained are of Tresca or Coulomb kind. The second case permits to show how it is possible to add various behaviour laws. The last case can model instabilities on the contact boundary.

\section{The mechanical problem}

The aim of this section is to present the mechanical problem and to give the notations used in the paper. We consider a body occupying an open bounded set $\Omega$ of $R^{3}$, with smooth boundary $\partial \Omega$, the three dimensional space is referred to the orthonormal 


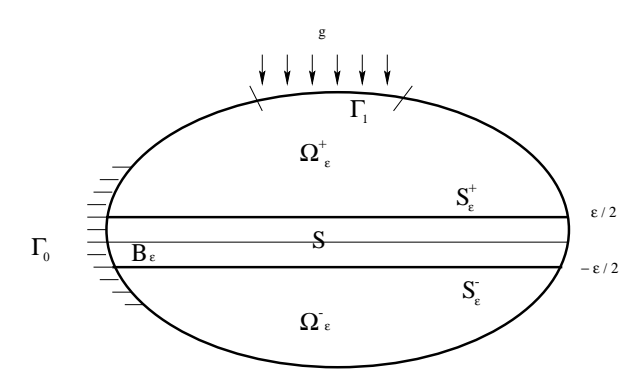

Figure 1: Geometry of the problem

frame $\left(O, x_{1}, x_{2}, x_{3}\right)$. This body is supposed to have a non-empty intersection $S$ with the plane $\left\{x_{3}=0\right\}$. Let $\varepsilon>0$, a parameter intended to tend to zero, we denote

$$
\begin{aligned}
B^{\varepsilon} & =\left\{x=\left(x_{1}, x_{2}, x_{3}\right) \in \Omega \text { such that }\left|x_{3}\right|<\frac{\varepsilon}{2}\right\}, \\
\Omega^{\varepsilon} & =\left\{x=\left(x_{1}, x_{2}, x_{3}\right) \in \Omega \text { such that }\left|x_{3}\right|>\frac{\varepsilon}{2}\right\}, \\
\Omega_{ \pm}^{\varepsilon} & =\left\{x=\left(x_{1}, x_{2}, x_{3}\right) \in \Omega \text { such that } \pm x_{3}>\frac{\varepsilon}{2}\right\}, \\
S_{ \pm}^{\varepsilon} & =\left\{x=\left(x_{1}, x_{2}, x_{3}\right) \in \Omega \text { such that } \pm x_{3}=\frac{\varepsilon}{2}\right\}, \\
\Omega_{ \pm} & =\left\{x=\left(x_{1}, x_{2}, x_{3}\right) \in \Omega \text { such that } \pm x_{3}>0\right\}, \\
S & =\left\{x=\left(x_{1}, x_{2}, x_{3}\right) \in \Omega \text { such that } x_{3}=0\right\}, \\
\Omega_{0} & =\Omega_{+} \cup \Omega_{-} .
\end{aligned}
$$

In the following, we suppose that $B^{\varepsilon}$ is the domain occupied by the joint and $\Omega^{\varepsilon}$ by the deformable bodies (see fig. 1), $\Omega^{0}$ being the geometrical limit of $\Omega^{\varepsilon}$. $S^{\varepsilon}$ is the interface between the adhesive and the adherents, and $S$ is the surface to which the adhesive tends geometrically. On the structure is applied a body force density $\varphi$ and a surface force density $g$ on $\Gamma_{1}$ a part of the boundary, $n$ denotes the external unit normal vector to $\Omega$. The part $\Gamma_{0}$ of the boundary is supposed to be embedded. The two bodies are supposed to be elastic and the joint will be considered successively as elastic, visco-elastic, elasto-plastic and pseudo-elastic. We denote by $\sigma^{\varepsilon}$ the stress tensor and $u^{\varepsilon}$ the displacement field. Under the small perturbations hypothesis, the strain tensor is written

$$
e_{i j}\left(u^{\varepsilon}\right)=\frac{1}{2}\left(\frac{\partial u_{i}^{\varepsilon}}{\partial x_{j}}+\frac{\partial u_{j}^{\varepsilon}}{\partial x_{i}}\right) .
$$

In the following, interface laws will be introduced between the bodies and the joint. We denote by $a_{i j k l}$ the elasticity parameters of the adherents. Thus, we have to solve the following problem

$$
\left(P_{\varepsilon}\right) \begin{cases}\text { Find }\left(u^{\varepsilon}, \sigma^{\varepsilon}\right) \text { such that : } & \\ \sigma_{i j, j}^{\varepsilon}=-\varphi_{i} & \text { in } \Omega \\ \sigma_{i j}^{\varepsilon}=a_{i j k h} e_{k h}\left(u^{\varepsilon}\right) & \text { in } \Omega^{\varepsilon} \\ + \text { behaviour laws } & \text { in } B^{\varepsilon} \\ u^{\varepsilon}=0 & \text { on } \Gamma_{0} \\ \sigma^{\varepsilon} n=g & \text { on } \Gamma_{1} \\ + \text { interface laws } & \text { on } S_{ \pm}^{\varepsilon}\end{cases}
$$


If $\psi$ is a function, we define by $\psi\left(x_{0}^{+}\right)$(resp. $\psi\left(x_{0}^{-}\right)$) the limit of $\psi(x)$ when $x$ tends to $x_{0}, x \geq x_{0}$ (resp. $x \leq x_{0}$ ). In order to define the interface laws between bodies and the joint, that is perfect adhesion, Signorini's law and Coulomb's law, we have to introduce some notations. We define the local frame $\left(n^{ \pm}, t^{ \pm}\right)$where $n^{ \pm}$is the external normal vector to $\Omega_{ \pm}^{\varepsilon}$. In the same way, we define $(n, t)$, where $n$ is external normal to $\Omega_{+}$. In these local basis, a vector $v$ is decomposed as

$$
\begin{aligned}
v\left(x_{1}, \pm \frac{\varepsilon}{2}\right) & =v_{N}\left(x_{1}, \pm \frac{\varepsilon}{2}\right) n^{ \pm}+v_{T}\left(x_{1}, \pm \frac{\varepsilon}{2}\right) \\
v\left(x_{1}, 0^{ \pm}\right) & =v_{N} n+v_{T}
\end{aligned}
$$

In the same way, the stress tensor $\sigma n$ on $S_{\varepsilon}$ or on $S$ is decomposed as

$$
\begin{aligned}
\sigma\left(x_{1}, \pm \frac{\varepsilon}{2}\right) n & =\sigma_{N} n^{ \pm}+\sigma_{T}, \\
\sigma\left(x_{1}, 0^{ \pm}\right) n & =\sigma_{N} n+\sigma_{T} .
\end{aligned}
$$

We define the relative displacements

$$
\begin{aligned}
{\left[v_{N}\right]_{ \pm} } & =v_{N}\left(x_{1}, \pm \frac{\varepsilon^{+}}{2}\right)+v_{N}\left(x_{1}, \pm \frac{\varepsilon^{-}}{2}\right), \\
{\left[v_{T}\right]_{ \pm} } & =v_{T}\left(x_{1}, \pm \frac{\varepsilon^{+}}{2}\right)+v_{T}\left(x_{1}, \pm \frac{\varepsilon^{-}}{2}\right), \\
{\left[v_{N}\right] } & =v_{N}\left(x_{1}, 0^{+}\right)+v_{N}\left(x_{1}, 0^{-}\right), \\
{\left[v_{T}\right] } & =v_{T}\left(x_{1}, 0^{+}\right)+v_{T}\left(x_{1}, 0^{-}\right) .
\end{aligned}
$$

In sections 4, 6 and 7, a perfect adhesion is supposed at the interface between the thin layer and the substrata

$$
\left[u^{\varepsilon}\right]_{ \pm}=0 \quad \text { on } S_{ \pm}^{\varepsilon}
$$

In section 5, we consider that there is contact with dry friction conditions between the bodies and the thin layer. The Signorini's law of unilateral contact and the Coulomb's law of dry friction are written in the case of monotone quasi-static loadings

$$
\begin{array}{rc}
\sigma_{N}^{\varepsilon} \leq 0 & \text { on } S_{ \pm}^{\varepsilon}, \\
{\left[u_{N}^{\varepsilon}\right]_{ \pm} \leq 0} & \text { on } S_{ \pm}^{\varepsilon}, \\
\sigma_{N}^{\varepsilon}\left[u_{N}^{\varepsilon}\right]_{ \pm}=0 & \text { on } S_{ \pm}^{\varepsilon}, \\
\left|\sigma_{T}^{\varepsilon}\right| \leq f\left|\sigma_{N}^{\varepsilon}\right| & \text { on } S_{ \pm}^{\varepsilon}, \\
\text { If }\left|\sigma_{T}^{\varepsilon}\right|<f\left|\sigma_{N}^{\varepsilon}\right| \text { then }\left[u_{T}^{\varepsilon}\right]_{ \pm}=0, & \\
\text { If }\left|\sigma_{T}^{\varepsilon}\right|<f\left|\sigma_{N}^{\varepsilon}\right| \text { then }\left[u_{T}^{\varepsilon}\right]_{ \pm}=-\zeta \sigma_{T} & \text { with } \zeta \geq 0,
\end{array}
$$

where $f$ is the friction coefficient.

\section{Mathematical background}

The idea of matched asymptotic expansions [4] (other techniques are given in [2, 5, $6,7]$ ) is to find two expansions of the displacement $u^{\varepsilon}$ and to the stress $\sigma^{\varepsilon}$ in powers of $\varepsilon$, that is, an external one in the bodies and an internal in the joint, and to connect 
these two expansions in order to obtain the same limit. In the following, we have considered a problem in dimension 2 in order to simplify the computations. The external expansion is a classical expansion in powers of $\varepsilon$

$$
\begin{aligned}
u^{\varepsilon}\left(x_{1}, x_{2}\right) & =u^{0}\left(x_{1}, x_{2}\right)+\varepsilon u^{1}\left(x_{1}, x_{2}\right)+\ldots, \\
\sigma_{i j}^{\varepsilon}\left(x_{1}, x_{2}\right) & =\sigma_{i j}^{0}\left(x_{1}, x_{2}\right)+\varepsilon \sigma_{i j}^{1}\left(x_{1}, x_{2}\right)+\ldots, \\
e_{i j}\left(u^{\varepsilon}\right)\left(x_{1}, x_{2}\right) & =e_{i j}^{0}+\varepsilon e_{i j}^{1}+\ldots, \\
e_{i j}^{l} & =\frac{1}{2}\left(\frac{\partial u_{i}^{l}}{\partial x_{j}}+\frac{\partial u_{j}^{l}}{\partial x_{i}}\right) .
\end{aligned}
$$

In the internal expansion, we proceed to a blow-up of the second variable. Let $y_{2}=\frac{x_{2}}{\varepsilon}$. The internal expansion gives

$$
\begin{aligned}
& u^{\varepsilon}\left(x_{1}, x_{2}\right)=v^{0}\left(x_{1}, y_{2}\right)+\varepsilon v^{1}\left(x_{1}, y_{2}\right)+\ldots, \\
& \sigma_{i j}^{\varepsilon}\left(x_{1}, y_{2}\right) \quad=\quad \varepsilon^{-1} \tau_{i j}^{-1}\left(x_{1}, y_{2}\right)+\tau_{i j}^{0}\left(x_{1}, y_{2}\right)+\varepsilon \tau_{i j}^{1}\left(x_{1}, y_{2}\right)+\ldots, \\
& e_{i j}\left(u^{\varepsilon}\right)\left(x_{1}, y_{2}\right)=\varepsilon^{-1} e_{i j}^{-1}+e_{i j}^{0}+\varepsilon e_{i j}^{1}+\ldots, \\
& e_{11}^{l}=\frac{\partial v_{1}^{l}}{\partial x_{1}}, \quad e_{22}^{l}=\frac{\partial v_{2}^{l+1}}{\partial y_{2}}, \quad e_{12}^{l}=\frac{1}{2}\left(\frac{\partial v_{2}^{l}}{\partial x_{1}}+\frac{\partial v_{1}^{l+1}}{\partial y_{2}}\right) .
\end{aligned}
$$

The third step of the method consists in the connection of the two expansions. In particular, we observe that when $\varepsilon$ tends to zero, $x_{2}$ tends to $0^{ \pm}$and $y_{2}$ tends to $\pm \infty$. The connection of the two expansions gives

$$
\begin{aligned}
v^{0}\left(x_{1}, \pm \infty\right) & =u^{0}\left(x_{1}, 0^{ \pm}\right), \\
\tau^{-1}\left(x_{1}, \pm \infty\right)=0, & \tau^{0}\left(x_{1}, \pm \infty\right)=\sigma^{0}\left(x_{1}, 0^{ \pm}\right) .
\end{aligned}
$$

The equilibrium equation at order -2 gives

$$
\frac{\partial \tau_{i 2}^{-1}}{\partial y_{2}}=0 \text {. }
$$

Thus, $\tau_{i 2}^{-1}$ does not depends on $y_{2}$. Due to the limit of $\tau_{i 2}^{-1}$ in $\pm \infty$, that is zero, we have

$$
\tau_{i 2}^{-1}=0
$$

The equilibrium equation at order -1 gives

$$
\frac{\partial \tau_{i 2}^{0}}{\partial y_{2}}=0 .
$$

Thus,

$$
\tau_{i 2}^{0}=\sigma_{i 2}^{0}\left(x_{1}, 0^{ \pm}\right)
$$

\section{Visco-elasticity}

\subsection{Kelvin-Voigt visco-elasticity}

We consider that the thin layer is visco-elastic and obeys to the Kelvin-Voigt's law of linear visco-elasticity. We consider a perfect adhesion between the bodies and the 
glue. The deformation tensor is splitted in two parts: an elastic part and an non-elastic one. The symbol () characterizes the time derivative. The behaviour law in the thin layer is given by

$$
\sigma_{i j}^{\varepsilon}=\lambda\left(e_{k k}+\theta_{\lambda} \dot{e}_{k k}\right) \delta_{i j}+2 \mu\left(e_{i j}+\theta_{\mu} \dot{e}_{i j}\right)
$$

where $\lambda$ and $\mu$ are the Lamé's coefficients. $\theta_{\lambda}$ and $\theta_{\mu}$ are two characteristic times. The asymptotic expansion gives:

$$
\begin{aligned}
\varepsilon^{-1} \tau_{i j}^{-1}+\tau_{i j}^{0}+\varepsilon^{1} \tau_{i j}^{1}+\ldots & =\lambda\left(\varepsilon^{-1}\left(e_{k k}^{-1}+\theta_{\lambda} \dot{e}_{k k}^{-1}\right)+e_{k k}^{0}+\theta_{\lambda} \dot{e}_{k k}^{0}\right. \\
& \left.+\varepsilon\left(e_{k k}^{1}+\theta_{\lambda} \dot{e}_{k k}^{1}\right)+\ldots\right) \delta_{i j} \\
& +2 \mu\left(\varepsilon^{-1}\left(e_{i j}^{-1}+\theta_{\mu} \dot{e}_{i j}^{-1}\right)+e_{i j}^{0}+\theta_{\mu} \dot{e}_{i j}^{0}\right. \\
& \left.+\varepsilon\left(e_{i j}^{1}+\theta_{\mu} \dot{e}_{i j}^{1}\right)+\ldots\right) .
\end{aligned}
$$

In the internal expansion, we observe that the important quantities are the ratio between the Lamé's coefficients (resp. the characteristic times) and the thickness of the thin layer. In order to simplify, we suppose that the two ratios associated to the Lamé's coefficients have a finite limit not equal to zero and that the ratios associated to the characteristic times have infinite limits. The other cases can be deduced easily by the techniques presented in the following. We denote by $\bar{\lambda}, \bar{\mu}$ the limits associated to the Lamé's coefficients. We obtain

$$
\tau_{i j}^{-1}=0, \quad \tau_{i j}^{0}=\bar{\lambda}\left(e_{k k}^{-1}+\theta_{\lambda} \dot{e}_{k k}^{-1}\right) \delta_{i j}+2 \bar{\mu}\left(e_{i j}^{-1}+\theta_{\mu} \dot{e}_{i j}^{-1}\right) .
$$

Thus, by integration in the interval $[-1 / 2,1 / 2]$ and using Equations (9), (10) and (14), we obtain

$$
\sigma_{12}^{0}=\bar{\mu}\left[u_{1}^{0}\right]+\bar{\mu} \theta_{\mu}\left[\dot{u}_{1}^{0}\right], \quad \sigma_{22}^{0}=(\bar{\lambda}+2 \bar{\mu})\left[u_{2}^{0}\right]+\left(\bar{\lambda} \theta_{\lambda}+2 \bar{\mu} \theta_{\mu}\right)\left[\dot{u}_{2}^{0}\right] .
$$

To summarize, the interface law is given by

$$
\sigma n=K_{L}[u]+K_{\theta}[\dot{u}] .
$$

The diagonal matrices $K_{L}$ and $K_{\theta}$ are deduced easily from (18). We observe that this interface law is valid for all the cases of coefficients variations. Particularly, if the limits of the ratios are infinite, the jumps in the former formulas are equal to zero (perfect adhesion). Conversely, if the coefficients are equal to zero, the stress tensor is equal to zero (perfect sliding).

\subsection{Maxwell visco-elasticity}

The thin layer is supposed to obey to the Maxwell's law of visco-elasticity. We consider a perfect adhesion between the bodies and the glue. The behaviour law in the thin layer is given by

$$
e\left(u^{\varepsilon}\right)=\frac{1+\nu}{E}\left(\dot{\sigma}_{i j}^{\varepsilon}+\frac{\sigma_{i j}^{\varepsilon}}{t_{1}}\right)-\frac{\nu}{E}\left(\dot{\sigma}_{k k}^{\varepsilon}+\frac{\sigma_{k k}^{\varepsilon}}{t_{2}}\right) \delta_{i j}
$$


$E$ is the Young's modulus and $\nu$ is the Poisson's ratio. $t_{1}$ and $t_{2}$ are two characteristic times. We use the same techniques that in the previous section. We assume that $t_{1}=$ $t_{2}$. The important value in the expansion is the ratio between the Young's modulus and the thickness of the thin layer. We denote $\bar{E}$ the limit of this ratio. We obtain

$$
\left[u_{1}^{0}\right]=\frac{1+\nu}{\bar{E}}\left(\dot{\sigma}_{12}^{0}+\sigma_{12}^{0} / t_{1}\right), \quad\left[u_{2}^{0}\right]=-\frac{1-\nu}{\bar{E} \nu}\left(\dot{\sigma}_{22}^{0}+\sigma_{22}^{0} / t_{1}\right)
$$

The interface law is written

$$
[u]=K_{Y} \dot{\sigma} n+K_{t} \sigma n \text {. }
$$

The matrices $K_{Y}$ and $K_{t}$ are deduced easily from Equation (21).

\subsection{Norton visco-elasticity}

The thin layer obeys to the non-linear Norton's law of visco-elasticity. We consider a perfect adhesion between the bodies and the glue. The behaviour law in the thin layer is given by

$$
\sigma_{i j}^{\varepsilon}=\lambda e_{k k} \delta_{i j}+2 \mu e_{i j}+\eta|\dot{e}|^{p-2} \dot{e}_{i j}
$$

where $\lambda$ and $\mu$ are the Lamé's coefficients and $\eta$ is the viscosity coefficient. $p$ is a scalar parameter. We use the same techniques that in previous sections. The characteristic coefficients in the expansions are the ratios between the Lamé's coefficients and the thickness of the thin layer and the ratio between the viscosity coefficient and the thickness at the power $p-1$. We denote $\bar{\lambda}, \bar{\mu}$ and $\bar{\eta}$ the limits of these ratios. We obtain

$$
\begin{aligned}
\sigma_{12}^{0} & =\bar{\mu}\left[u_{1}^{0}\right]+\bar{\eta} / 2 \int_{-\frac{1}{2}}^{\frac{1}{2}}|\dot{e}|^{p-2} \frac{\partial \dot{v}_{1}^{0}}{\partial y_{2}} d y_{2}, \\
\sigma_{22}^{0} & =(\bar{\lambda}+2 \bar{\mu})\left[u_{2}^{0}\right]+\bar{\eta} \int_{-\frac{1}{2}}^{\frac{1}{2}}|\dot{e}|^{p-2} \frac{\partial \dot{v}_{2}^{0}}{\partial y_{2}} d y_{2} .
\end{aligned}
$$

We have to solve two differential equations. The stresses $\sigma_{12}^{0}$ and $\sigma_{22}^{0}$ depend only on $x_{1}$, thus $\frac{\partial \dot{v}_{1}^{0}}{\partial y_{2}}$ and $\frac{\partial \dot{v}_{2}^{0}}{\partial y_{2}}$ do not depend on $y_{2}$. The displacements $\dot{v}_{1}^{0}$ and $\dot{v}_{2}^{0}$ depend linearly on $y_{2}$. We deduce by integration

$$
\begin{aligned}
\sigma_{12}^{0} & =\bar{\mu}\left[u_{1}^{0}\right]+\bar{\eta} / 2\left|[\dot{u}] \otimes_{s} n\right|^{p-2}\left[\dot{u}_{1}^{0}\right], \\
\sigma_{22}^{0} & =(\bar{\lambda}+2 \bar{\mu})\left[u_{2}^{0}\right]+\bar{\eta}\left|[\dot{u}] \otimes_{s} n\right|^{p-2}\left[\dot{u}_{2}^{0}\right],
\end{aligned}
$$

where the symbol $\otimes_{s}$ denotes the symmetric tensorial product. To summarize the interface law is written

$$
\sigma n=K_{L}[u]+K_{\eta}\left|[\dot{u}] \otimes_{s} n\right|^{p-2}[\dot{u}] .
$$

The matrices $K_{L}$ and $K_{\eta}$ are deduced easily from Equation (26). 


\subsection{Incompressibility}

We consider a perfect adhesion between the bodies and the glue. The incompressibility condition is written in the quasi-static case

$$
\text { divu }=0 .
$$

The former equation becomes

$$
\varepsilon^{-1} \frac{\partial u_{2}}{\partial y_{2}}+\frac{\partial u_{1}}{\partial x_{1}}+\ldots=0
$$

Thus,

$$
\frac{\partial u_{2}}{\partial y_{2}}=0
$$

and

$$
\left[u_{2}\right]=0
$$

We obtain the law of bilateral contact

$$
\left[u_{N}\right]=0
$$

\section{Frictional contact at the interface thin layer-substrata}

\subsection{Mathematical results}

In this section, we consider that the contact obeys to the laws of Signorini and Coulomb. The thin layer is supposed to be elastic. We examine only the case $\bar{\lambda}=0$ and $0<\bar{\mu}<\infty$. The other cases can be deduced by the same arguments. In this case $\tau_{11}^{0}=0, \tau_{12}^{0}=\bar{\mu} \frac{\partial v_{1}^{0}}{\partial y_{2}}, \tau_{22}^{0}=2 \bar{\mu} \frac{\partial v_{2}^{0}}{\partial y_{2}}$. Thus, by integration and due to the continuity conditions, we have

$$
\begin{aligned}
& \sigma_{12}^{0}\left(x_{1}, 0\right)=\bar{\mu}\left(v_{1}^{0}\left(x_{1}, \frac{1}{2}^{-}\right)-v_{1}^{0}\left(x_{1},-\frac{1}{2}^{+}\right)\right), \\
& \sigma_{22}^{0}\left(x_{1}, 0\right)=2 \bar{\mu}\left(v_{2}^{0}\left(x_{1}, \frac{1}{2}^{-}\right)-v_{2}^{0}\left(x_{1}, \frac{1}{2}^{+}\right)\right) .
\end{aligned}
$$

First, we examine the normal components. The contact law on surfaces $S_{ \pm}^{\varepsilon}$ gives

$$
\begin{aligned}
v_{2}^{0}\left(x_{1}, \frac{1}{2}^{+}\right)-v_{2}^{0}\left(x_{1}, \frac{1}{2}^{-}\right) & \geq 0 \\
v_{2}^{0}\left(x_{1},{-\frac{1}{2}^{-}}^{-}\right)-v_{2}^{0}\left(x_{1},-\frac{1}{2}^{+}\right) & \geq 0 .
\end{aligned}
$$

If $\sigma_{22}^{0}\left(x_{1}, 0\right)=0\left(\sigma_{N}^{0}=0\right)$ then Equations (32) and (33) give

$$
\left[u_{N}^{0}\right] \leq 0
$$

If $\sigma_{22}^{0}\left(x_{1}, 0\right)<0\left(\sigma_{N}^{0}<0\right)$ then the contact law and (32) give

$$
\sigma_{22}^{0}\left(x_{1}, 0\right)=2 \bar{\mu}\left(v_{2}^{0}\left(x_{1}, \frac{1}{2}^{+}\right)-v_{2}^{0}\left(x_{1},-\frac{1}{2}^{-}\right)\right) .
$$




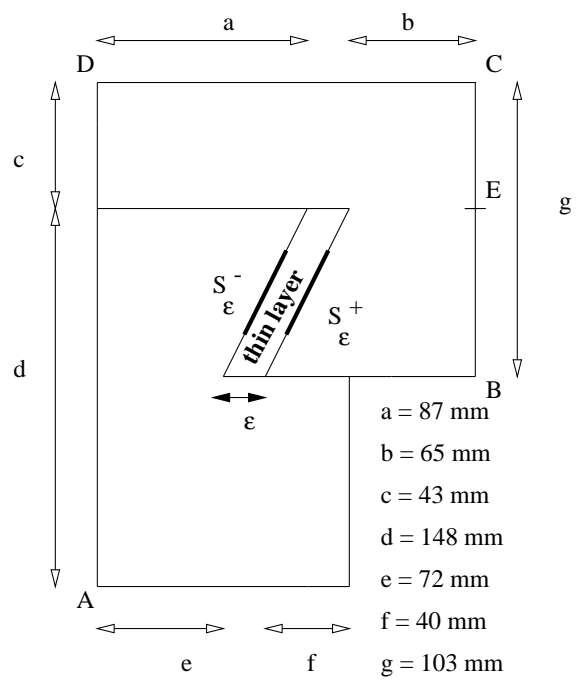

Figure 2: A dovetail assembly

Then, we examine the tangential components. If $\left|\sigma_{12}^{0}\right|<f\left|\sigma_{22}^{0}\right|\left(\left|\sigma_{T}^{0}\right|<f\left|\sigma_{N}^{0}\right|\right)$ then the friction law and (32) give

$$
\sigma_{12}^{0}\left(x_{1}, 0\right)=\bar{\mu}\left(v_{1}^{0}\left(x_{1}, \frac{1}{2}^{+}\right)-v_{1}^{0}\left(x_{1},-\frac{1}{2}^{-}\right)\right) .
$$

If $\left|\sigma_{12}^{0}\right|=f\left|\sigma_{22}^{0}\right|\left(\left|\sigma_{T}^{0}\right|=f\left|\sigma_{N}^{0}\right|\right)$ then the friction law and (32) give

$$
\begin{aligned}
v_{1}^{0}\left(x_{1}, \frac{1}{2}^{+}\right)-v_{1}^{0}\left(x_{1}, \frac{1}{2}^{-}\right) & =\zeta_{1} \sigma_{12}^{0}\left(x_{1}, 0\right), \\
v_{1}^{0}\left(x_{1},-\frac{1}{2}^{-}\right)-v_{1}^{0}\left(x_{1},-\frac{1}{2}^{+}\right) & =\zeta_{2} \sigma_{12}^{0}\left(x_{1}, 0\right), \\
v_{1}^{0}\left(x_{1}, \frac{1}{2}^{+}\right)-v_{1}^{0}\left(x_{1},-\frac{1}{2}^{-}\right) & =\left(\frac{1}{\bar{\mu}}+\zeta_{1}+\zeta_{2}\right) \sigma_{12}^{0}\left(x_{1}, 0\right), \\
{\left[u_{T}^{0}\right] } & =-\left(\frac{1}{\bar{\mu}}+\zeta\right) \sigma_{T}^{0}\left(x_{1}, 0\right) .
\end{aligned}
$$

To summarize, we obtain the following compliance contact law

$$
\begin{gathered}
\sigma_{N}^{0}=-2 \bar{\mu}\left[u_{N}^{0}\right]^{+}, \\
\left|\sigma_{T}^{0}\right| \leq f\left|\sigma_{N}^{0}\right|, \\
\text { If }\left|\sigma_{T}^{0}\right|<f\left|\sigma_{N}^{0}\right| \text { then } \sigma_{T}^{0}=-\bar{\mu}\left[u_{T}^{0}\right], \\
\text { If }\left|\sigma_{T}^{0}\right|=f\left|\sigma_{N}^{0}\right| \text { then }\left[u_{T}^{0}\right]=-\zeta \sigma_{T}^{0}, \zeta \geq 0 .
\end{gathered}
$$

\subsection{Numerical validations}

In this part, we proceed as in former papers [1]. The example of a dovetail assembly is treated (figure 2). The idea of the numerical study is to verify that the numerical results are coherent with the theory (validation) and to determine the thickness of the layer for which the limit interface law could be considered as valid (quantization). Thus, we solve numerically the initial problem with a thin layer having decreasing values of the thickness and stiffness.

The coefficients $\varepsilon, \lambda$ and $\mu$ are chosen smaller and smaller. After that, numerical 


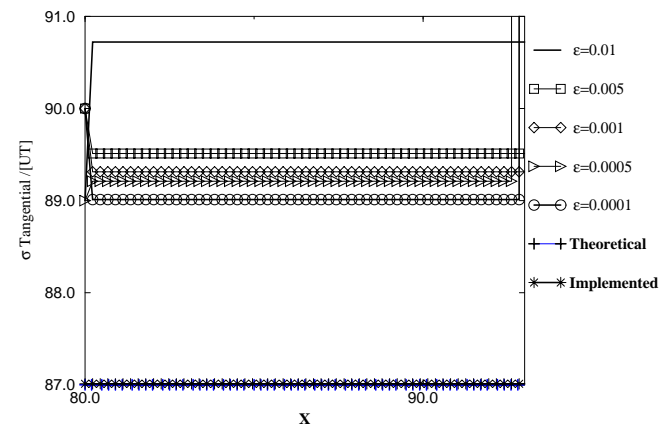

Figure 3: Tangential rate (stress-displacement) for a decreasing thickness

results are compared with theoretical results. In order to simplify the computations, we suppose that Lamé's coefficients of the joint are given by $\lambda=\varepsilon^{\gamma} \lambda, \gamma \geq 0$ and $\mu=\varepsilon^{\delta} \mu, 0 \leq \delta<2$. We solve the problem with decreasing values of $\varepsilon$, $\varepsilon$ will be equal to $1.10^{-2}, 5.10^{-3}, 1.10^{-3}, 5.10^{-4}, 1.10^{-4}$. In order to study the results, we compute the displacement fields $\left(u_{N}, u_{T}\right)$ and the stress vector $\left(\sigma_{N}, \sigma_{T}\right)$ on $S_{\varepsilon}^{ \pm}$for the initial problem (resp. on $S$ for the limit problem) for the nine couples $(\lambda, \mu)$. After that, we compare theoretical curves and numerical curves for stick or sliding nodes. We obtain the same kind of results. The limit law can considered as valid for a thickness of the thin layer smaller than $10^{-3} \mathrm{~mm}$.

\subsection{Numerical algorithm}

The previous contact problem is written Find $\rho$ fixed point of the application $\rho \longrightarrow$ $f\left|\sigma_{N}(u(\rho))\right|$, where $u=u(\rho)$ is solution of

$$
\left\{\begin{array}{l}
\text { Find } u \in V \text { such that }: \\
J(u) \leq J(v),
\end{array} \forall v \in V,\right.
$$

with $J(v)=\frac{1}{2} A(v, v)-L(v)+\int_{S} \phi\left(v_{N}\right) d s+\int_{S} \psi\left(v_{T}\right) d s ., \phi$ is given by

$$
\phi\left(v_{N}\right)= \begin{cases}0 & \text { if } v_{N}<0 \\ \frac{K_{N}}{2}\left(v_{N}\right)^{2} & \text { if } v_{N} \geq 0 .\end{cases}
$$

$\psi$ is given by

$$
\psi\left(v_{T}\right)= \begin{cases}-\rho v_{T}-\frac{\rho^{2}}{2 K_{T}} & \text { if } v_{T}<\frac{-\rho}{K_{T}}, \\ \frac{1}{2} K_{T} \cdot\left(v_{T}\right)^{2} & \text { if }\left|v_{T}\right| \leq \frac{\rho}{K_{T}}, \\ \rho v_{T}-\frac{\rho^{2}}{2 K_{T}} & \text { if } v_{T}>\frac{\rho}{K_{T}} .\end{cases}
$$

The Problem is discretized by a finite element method formulated in displacements. Usually, we use $P 1$ finite elements (triangles with three nodes and six degrees of 
freedom) or $Q 1$ (quadrangles with four nodes and eight degrees of freedom). We have to minimize a functional still noted $J$ in $R^{2 N P}$. We denote: NP the total number of nodes, NC the number of contact nodes, $I_{N}$ the indices of normal components of contact nodes, $I_{T}$ the indices of tangential components of contact nodes, $A$ the stiffness matrix with coefficient $a_{i j}$ and $B$ the stiffness matrix to the surface terms with coefficient $b_{i j}$. The relaxation method consists in finding the solution of problem 2 solving a sequence of minimization problems in $R^{2 N P}$

$$
\left\{\begin{array}{l}
\text { Find } u_{i}^{n+\frac{1}{2}} \text { such that } \forall v \in R^{2 N P} \\
J\left(u_{1}^{n+1}, \ldots, u_{i-1}^{n+1}, u_{i}^{n+\frac{1}{2}}, u_{i+1}^{n}, \ldots, u_{2 N P}^{n}\right) \leq J\left(u_{1}^{n+1}, \ldots, u_{i-1}^{n+1}, v, u_{i+1}^{n}, \ldots, u_{2 N P}^{n}\right)
\end{array}\right.
$$

We denote by $\omega$ the relaxation coefficient. In the first time, we are interested by the normal components.

- $i \in I_{N}$

The algorithm is written

$$
\left\{\begin{array}{l}
u_{i}^{n+\frac{1}{2}}=\frac{1}{d_{i i}^{n+\frac{1}{2}}}\left(\varphi_{i}-\sum_{j=1}^{i-1} d_{i j}^{n+1} u_{j}^{n+1}-\sum_{j=i+1}^{2 N P} d_{i j}^{n} u_{j}^{n}\right) \\
\text { with } \\
d_{i j}^{n}=\left\{\begin{array}{l}
a_{i j}+\gamma\left(u_{j}^{n}\right) b_{i j} \quad \text { if } j \in I_{N} \\
a_{i j}+\eta\left(u_{j}^{n}\right) b_{i j} \quad \text { if } j \in I_{T} \\
a_{i j} \quad \text { otherwise }
\end{array}\right. \\
\gamma(u)= \begin{cases}0 & \text { if } u \leq 0 \\
1 & \text { if } u>0\end{cases} \\
\text { and } \eta(u)= \begin{cases}0 & \text { if }|u|>\frac{\rho}{K_{T}} \\
1 & \text { if }|u| \leq \frac{\rho}{K_{T}}\end{cases} \\
u_{i}^{n+1}=(1-\omega) u_{i}^{n}+\omega u_{i}^{n+\frac{1}{2}}
\end{array}\right.
$$

For tangential components, we have

- $i \in I_{T}$

First, the fixed point problem is considered $\rho_{i}^{l+1}=f\left|\sigma_{N}\left(u\left(\rho_{i}^{l}\right)\right)\right|$ and we write

$$
\left\{\begin{array}{l}
u_{i}^{n+\frac{1}{2}}=\frac{1}{d_{i i}^{n+\frac{1}{2}}}\left(\varphi_{i}-\sum_{j=1}^{i-1} d_{i j}^{n+1} u_{j}^{n+1}-\sum_{j=i+1}^{2 N P} d_{i j}^{n} u_{j}^{n}-\theta\left(u_{i}^{n+\frac{1}{2}}\right) \cdot \rho_{i}^{l}\right) \\
\text { with } \\
\theta(u)= \begin{cases}-1 & \text { if } u<\frac{-\rho}{K_{T}} \\
1 & \text { if } u>\frac{\rho}{K_{T}} \\
0 & \text { if }|u| \leq \frac{\rho}{K_{T}}\end{cases} \\
u_{i}^{n+1}=(1-\omega) u_{i}^{n}+\omega u_{i}^{n+\frac{1}{2}}
\end{array}\right.
$$

\subsection{An academic test}

In this paragraph the algorithm is tested and validated. We have chosen a benchmark studied by the group "Validation of computer codes" of French Research Groupment 


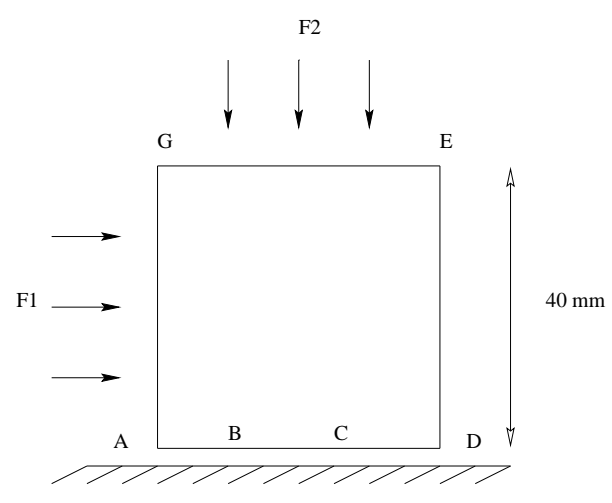

Figure 4: The example of a long bar

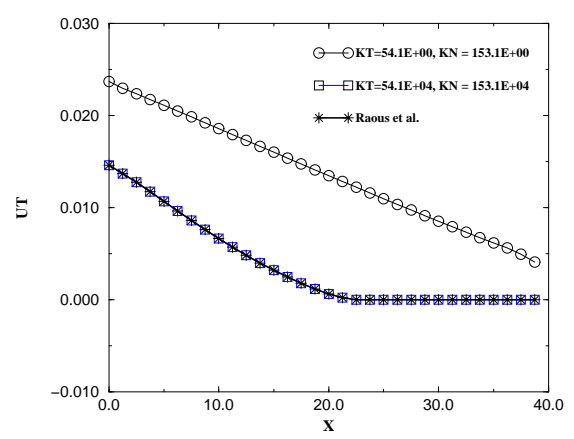

Figure 5: Tangential contact force for various coefficient $K_{N}=\bar{\lambda}+2 \bar{\mu}, K_{T}=\bar{\mu}$

"Large Deformations and Damage" ([9], figure 4). We work in plane strains and we consider a long bar with square section with Young's modulus $E=130 \mathrm{Gpa}$ and Poisson's ratio $\nu=0.2$. The contact zone (interface law) corresponds to the part $A D$ with a friction coefficient equal to $f=1 . u_{1}=0$ on $D E$ and $u_{1}=u_{2}=0$ at point $D$. We impose a loading $F 1=10 \mathrm{daN} / \mathrm{mm}^{2}$ on $A G$ and $F 2=-5 \mathrm{daN} / \mathrm{mm}^{2}$ on $G E$. In the finite element method, the contact zone is discretized by 32 nodes. For different values of $\bar{\mu}$ and $\bar{\lambda}$ the variations of contact status are studied (figure 5). We compare the results with [9] (Signorini-Coulomb laws). We observe that for $\bar{\lambda}=4.510^{+8}$ and $\bar{\mu}=5.410^{+8}$, we find the same results that in [9]. These coefficients correspond to the limit case (Signorini-Coulomb). The decreasing of $\bar{\mu}$ corresponds to the increasing of the stick zone $(13,15$ and 32 nodes). Nevertheless, the coefficients have a strong influence on the tangential displacements. A low value of the coefficient $K_{T}$ implies an increasing of the tangential displacement. The nodes are sliding without reaching the value of the Coulomb's sliding limit. 


\section{Mohr-Coulomb elasto-plasticity}

\subsection{Mathematical results}

We consider a perfect adhesion between the bodies and the glue. The deformation is decomposed in an elastic part and in a plastic one. The thin layer obeys to the Mohr-Coulomb's law of elasto-plasticity, that is

$$
\begin{aligned}
e\left(u^{\varepsilon}\right) & =e^{e}+e^{p}, \\
\left|\sigma_{t}\right| & \leq C-\tan \Omega\left|\sigma_{n}\right|, \\
\sigma_{i j}^{\varepsilon} & =\lambda e_{k k} \delta_{i j}+2 \mu e_{i j}, \\
\dot{e}^{p} & =k \bar{n} \otimes_{s} \bar{t},
\end{aligned}
$$

where $\Omega$ is the friction angle, $C$ is the cohesion strength, $\sigma_{n}$ and $\sigma_{t}$ are respectively the normal and the tangential stresses; we denote by $\bar{n}$ and $\bar{t}$ the normal and tangential directions of the face corresponding to the plastic yield, $k$ is a non-negative parameter which is equal to zero in the elastic zone. We proceed as in the former sections. We consider the same case than in section 3. The internal expansion gives

$$
\tau_{12}^{0}=\bar{\mu}\left(\frac{\partial u_{1}}{\partial y_{2}}-e_{12}^{p}\right), \quad \tau_{22}^{0}=(\bar{\lambda}+2 \bar{\mu})\left(\frac{\partial u_{2}}{\partial y_{2}}-e_{22}^{p}\right) .
$$

Before the beginning of the plastification, we find the elasto-static case.

$$
\sigma n=K_{L}[u]
$$

When the plastic yield is reached, we obtain

$$
\tau_{12}^{0}=\bar{\mu}\left(\left[u_{1}^{0}\right]-\left[u_{1}^{p}\right]\right), \quad \tau_{22}^{0}=(\bar{\lambda}+2 \bar{\mu})\left(\left[u_{2}^{0}\right]-\left[u_{2}^{p}\right]\right) .
$$

The plastic flow is given by

$$
\left[\dot{u}^{p}\right]=k \bar{t}
$$

with $\left[\dot{u}_{i}^{p}\right]=\int_{-1 / 2}^{1 / 2} \dot{e}_{i 2}^{-1, p} d y_{2}$. The limit problem is quite different in this non-linear case. The plastic yield and the plastic flow depend on a local problem because they do not depend on the stress vector but on the total stress tensor. In order to determine the limit and the direction of sliding, we have to solve the following elasto-plastic problem in $S \times[-1 / 2,1 / 2]$

$$
\begin{aligned}
\frac{\partial \tau_{i 2}^{0}}{\partial y_{2}} & =0, \\
e^{-1} & =e^{e}+e^{p} \\
\left|\tau_{t}^{0}\right| & \leq C-\tan \Omega\left|\tau_{n}^{0}\right|, \\
\tau_{i j}^{0} & =\lambda e_{k k}^{-1} \delta_{i j}+2 \mu e_{i j}^{-1}, \\
\dot{e}^{p} & =k \bar{n} \otimes_{s} \bar{t} .
\end{aligned}
$$

Due to the fact that the solution does not depend on the thickness, we have to solve only a "one-dimensional problem". 


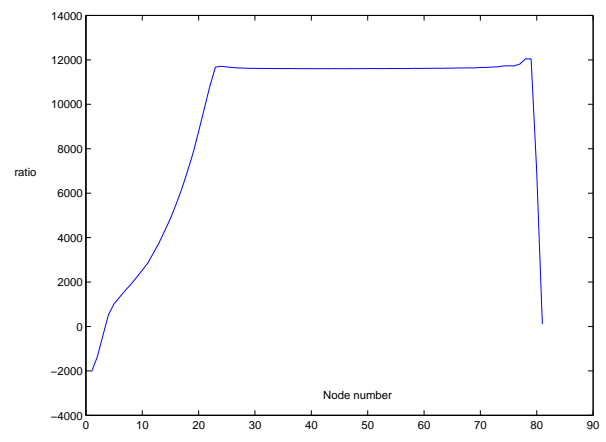

Figure 6: Ratio tangential force-tangential displacement on the contact zone : beginning of the plastification in the left part of the boundary.

Remarks a) If the direction of flow is equal to $x_{2}$, we find the Coulomb's friction law; b) In order to suppress the local problem, the unknown direction can be approximated by the tangential direction $x_{2}$; c) If the Mohr-Coulomb's law is replaced by a simplified law, that is the direction is given, the local problem is easier to solve.

\subsection{Numerical validations}

The example presented in section 5.4 is treated with an elasto-plastic thin layer. The results are shown in figure 6 . We observe that the theoretical law is verified with a good agreement. The ratio thickness of the thin layer - dimension of the body is equal to $1 / 100$.

\section{Some remarks on non-monotone behavior}

\subsection{Mathematical results and limitations}

We consider a perfect adhesion between the bodies and the glue. The behaviour of the material is non-monotone, that is

$$
\begin{aligned}
\sigma_{i j} & =\lambda e_{k k} \delta_{i j}+2 \mu e_{i j}+\alpha(T) \chi_{i j}, \text { if } \alpha(T) \chi_{i j} e_{i j} \leq-C(T) \\
\sigma_{i j} & =\lambda e_{k k} \delta_{i j}+2 \mu e_{i j}, \text { if }\left|\alpha(T) \chi_{i j} e_{i j}\right| \leq C(T) \\
\sigma_{i j} & =\lambda e_{k k} \delta_{i j}+2 \mu e_{i j}-\alpha(T) \chi_{i j}, \text { if } \alpha(T) \chi_{i j} e_{i j} \geq C(T),
\end{aligned}
$$

where $T$ is the temperature, $C(T)$ and $\alpha(T)$ are $\mathrm{n}$ the non-negative parameters of the law, and $\chi$ is a given orientation tensor. Using the same techniques and the same notations than in the previous sections, the internal expansion, in the case

$$
\alpha(T) \chi_{i j} e_{i j} \geq C(T)
$$


leads to

$$
\tau_{12}^{0}=\bar{\mu} \frac{\partial u_{1}^{0}}{\partial y_{2}}-\bar{\alpha} \chi_{12}, \quad \tau_{22}^{0}=(\bar{\lambda}+\bar{\mu}) \frac{\partial u_{2}^{0}}{\partial y_{2}}-\bar{\alpha} \chi_{22},
$$

where $\bar{\alpha}$ is the limit of the ratio between the coefficient $\alpha(T)$ and the thickness at the power $\eta<1$. In the following, $\bar{C}$ will denote the ratio between $C(T)$ and the same power of the thickness. By integration, we obtain

$$
\sigma_{12}^{0}=\bar{\mu}\left[u_{1}^{0}\right]-\bar{\alpha} \chi_{12}, \quad \sigma_{22}^{0}=(\bar{\lambda}+\bar{\mu})\left[u_{1}^{0}\right]-\bar{\alpha} \chi_{22} .
$$

Thus, we obtain an interface law written

$$
\text { If } \bar{\alpha} \chi_{i 2}\left[u_{i}\right] \geq \bar{C} \quad \text { then } \quad \sigma n=K_{L}[u]-\bar{\alpha} \chi_{.2} \text {. }
$$

\subsection{A 1D example}

The previous computations are not totally true because the problem does not have an unique solution. In fact, in the asymptotic expansion a particular solution is chosen. In this paragraph we treat the example of a bar of length $l$ occupying the interval $] 0, l[$. In the interval $] 0, \varepsilon l[$, the material has a behavior as in the previous section with a rigidity $k^{\prime}$; in the interval $] \varepsilon l, l[$, the material is elastic with a rigidity $K$. We suppose that a given displacement $\delta>0$ is applied at the extremity $x=l$. The bar is embedded at the extremity $x=0$. The adhesion is perfect for $x=\varepsilon l$. In this case, it is possible to show (after long computations !) that a global minimizer of the energy verifies

$$
\begin{aligned}
u_{a}(x) & = & \frac{E}{k^{\prime}} x \text { if } 0 \leq x \leq \varepsilon S, \\
u_{a}(x) & = & \frac{E+\alpha}{k^{\prime}}(x-\varepsilon S) \text { if } \varepsilon S \leq x \leq \varepsilon l, \\
u_{b}(x) & = & \frac{E^{K}}{K}(x-l)+\delta \text { if } \varepsilon l \leq x \leq l, \\
\varepsilon S & = & \varepsilon l\left(1+\frac{E}{\alpha}-\frac{E k^{\prime}}{\alpha K} l-\frac{k^{\prime}}{\alpha} \delta\right), \\
E & = & \frac{k^{\prime}}{\varepsilon l} \frac{K}{K_{\varepsilon}} \text { if } 0 \leq \delta \leq \delta_{0}, \\
E & = & \left.E_{m}=k^{\prime} \frac{C}{\alpha}-\frac{\alpha}{2}\right) \text { if } \delta_{0} \leq \delta \leq \delta_{1}, \\
E & = & \frac{k^{\prime}}{\varepsilon l}\left(\frac{K}{K_{\varepsilon}}+\frac{\alpha l}{K_{\varepsilon}}\right) \text { if } \delta>\delta_{1}, \\
\delta_{0}=\sigma_{m} l K_{\varepsilon}^{-1}, & \delta_{1}=\delta_{0}+l \varepsilon \frac{\alpha}{K}, & K_{\varepsilon}^{-1}=\left(\frac{1-\varepsilon}{K}+\frac{\varepsilon}{k^{\prime}}\right),
\end{aligned}
$$

$u_{a}$ (resp. $u_{b}$ ) is the displacement field in the interval $] 0, \varepsilon l[$ (resp. $] \varepsilon l, l[$ ). Thus, using the usual notations, the asymptotic law is given by

$$
\begin{array}{rll}
\sigma n & = & \bar{k}^{\prime}[u], \text { if } 0 \leq \delta \leq \delta_{0}^{0}, \\
\sigma n & = & \frac{l}{K}(\delta-[u]) \text { if } \delta_{0}^{0} \leq \delta \leq \delta_{1}^{1}, \\
\sigma n & = & \bar{k}^{\prime}[u]-\bar{\alpha}, \text { if } \delta>\delta_{1}^{0}, \\
\delta_{0}^{0}=\sigma_{m} \bar{K}^{-1}, & \delta_{1}^{0}=\delta_{0}^{0}+l \frac{\bar{\alpha}}{\bar{k}^{\prime}}, & \bar{K}^{-1}=\left(\frac{1}{K}+\frac{1}{\bar{k}^{\prime}}\right) .
\end{array}
$$

We observe that this law is quite different that the law obtained in subsection 7.1. 


\section{Conclusion: Rheological interpretation}

If one observes the mechanical model obtained in section 5, one can give a rheological interpretation to this model. The normal part of the model (contact law) can be interpreted as the sequence between a spring and a stop. At the limit the behavior of the thin layer is conserved, a spring corresponds to the elastic part of the model, and the stop corresponds to the non-linear part of the model. In fact, the sequence elasticityunilateral contact becomes at the limit the sequence spring-stop. This result is quite general. As an example, a thin layer made by two thin layers with different stiffnesses becomes a sequence of two springs with different rigidities. In the same way, the tangential part of the model can be seen as a sequence between a spring corresponding to the elasticity of the thin layer and a skidding block corresponding to the friction on the surface between the adherents and the adhesive.

\section{References}

[1] F. Lebon, A. Ould Khaoua, C. Licht "Numerical study of soft adhesively bonded joints in finite elasticity”, Computational Mechanics, 21, 134-140, 1998.

[2] C. Licht "Comportement asymptotique d'une bande dissipative mince de faible rigidité”, Comptes Rendus Académie des Sciences Paris, Série I, 322, 295-300, 1992.

[3] F. Lebon, C. Licht, F. Zaittouni "Asymptotic modelling of interfaces taking into account contact conditions, Part 1 Asymptotic expansions and numerical implementation”, International Journal of Solids and Structures, to appear, 2002.

[4] J. Sanchez-Hubert, E. Sanchez-Palencia "Introductions aux méthodes asymptotiques et à l'homogénéisation”, Masson, Paris, 1992.

[5] P. Suquet "Discontinuities and plasticity", in Nonsmooth mechanics and applications, J.J. Moreau and P.D. Panagiotopoulos Eds., CISM Courses and Lectures, 302, Springer-Verlag, 279-340, 1988.

[6] G. Bayada, K. Lhalouani "Asymptotic and numerical analysis for unilateral contact problem with Coulomb's friction between an elastic body and a thin elastic soft layer", Asymptotic Analysis, 25, 329-362, 2001.

[7] F. Zaittouni, F. Lebon, C. Licht "Etude théorique et numérique du comportement d'un assemblage de plaques”, Comptes Rendus Académie des Sciences Paris, Série Mécanique, 330, 359-364, 2002.

[8] J. Lemaitre, J.L. Chaboche "Mécanique des matériaux solides", Dunod, Paris, 1985.

[9] M. Raous, P. Chabrand, F. Lebon "Numerical methods for solving unilateral contact problem with friction", Journal of Theoretical and Applied Mechanics, 7, 111-128, 1988. 